\title{
Xyloglucan in der Wundheilung
}

Häufig werden Hydrokolloidbildner auf Basis von Polysacchariden in der klinischtherapeutischen Praxis zur Herstellung von sog. hydroaktiven Wundverbänden genutzt. Die hierfür verwendeten Polysaccharide (z.B. Alginate, Cellulosederivate o.Ä.) sollen ein idealfeuchtes Wundklima schaffen, das Austrocknen des regenerierenden Bindegewebes und der Epithelzellen verhindern, Wundsekret aufsaugen und auch mechanischen Wundschutz binden. In einer kürzlich erschienen Publikation wurde der hochmolekulare Gelbildner Xyloglucan (isoliert aus den Samen der Kapuzinerkresse, Tropaeolum majus L.) beschrieben, der als ein relativ starker Induktor der zellulären terminalen Differenzierung humaner, primärer Keratinozyten identifiziert wurde. Dies bedeutet, dass das entsprechende Polymer aktiv in die Wundheilung und zelluläre Regeneration eingreift und die proliferierenden Keratinozyten in die terminale Differenzierung triggert.

Hierfür wird das intrazelluläre „signalling“ durch Xyloglucan umgeschaltet, und zwar von Signalwegen, die mitotische Zellteilung bewirken hin zu zellulären Prozessen, welche die Proliferation stoppen und die Zelle in die terminale Differenzierung bewegen. Dies wiederum geht mit der Bildung von differenzierungstypischen Proteinen und Gerüstsubstanzen, wie z.B. Zytokeratin K10, Involucrin, Loricrin, Filagrin etc., einher. Hierdurch bilden sich die hauttypischen Barrierestrukturen solcher Zellen aus. D.h., solche Differenzierungsvorgänge stellen sehr wichtige Schritte in den letzten Phasen der Wundheilung und Hautregeneration dar.

Die Autoren der Publikation zeigten, dass Xyloglucan tatsächlich die Zellproliferation stoppt, die Bildung der Diffe-

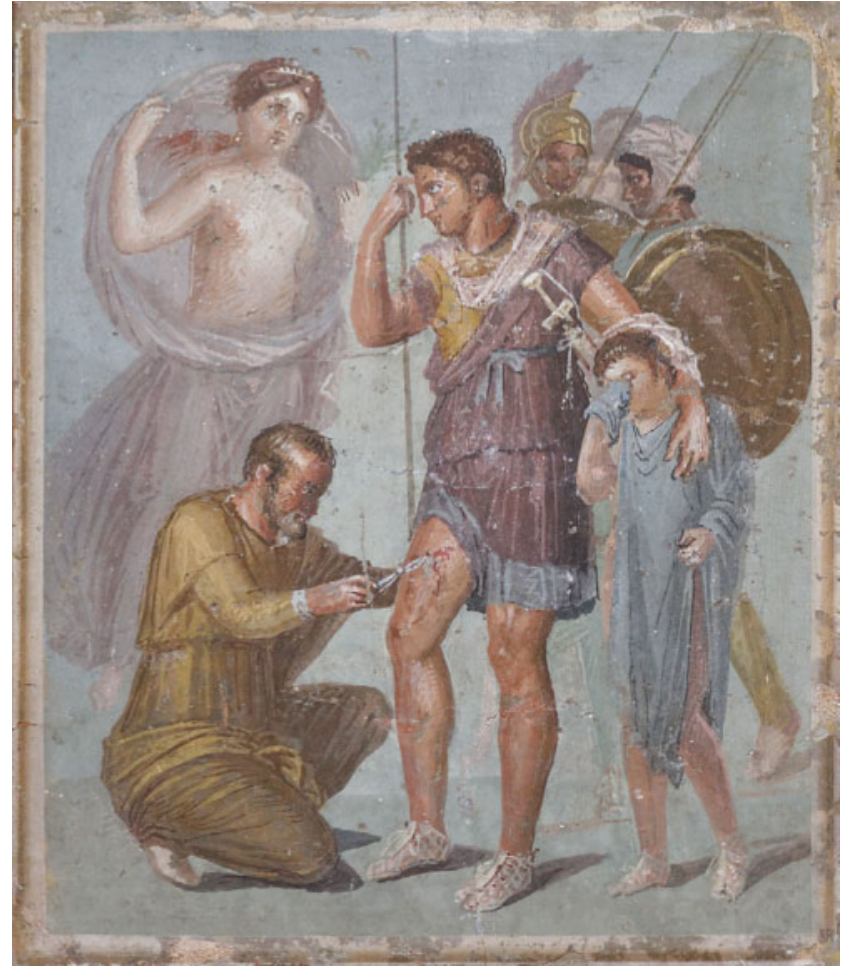

Als es noch keine Hydrokolloid-Therapie gab: Wundversorgung des Aeneas durch den Arzt Japix. Fresko, 1. Jh. n. Chr. Archäologisches Nationalmuseum Neapel. (c) Marie-Lan Nguyen

renzierungsmarker induziert und der Differenzierungsprozess dann kontrolliert abläuft. Interessant ist aber auch der zugrunde liegende Wirkmechanismus: Xyloglucan inhibiert auf molekularer Ebene die Phosphorylierung des epidermalen Wachstumsfaktor-Rezeptors EGFR auf der Außenseite der Keratinozyten, wodurch das interzelluläre „signalling“ zur mitotischen Zellteilung über den MAPK/ ERK-Signalweg reduziert wird. Der am Ende dieses Signalwegs stehende Transkriptionsfaktor CREB wird dadurch in seiner Aktivität gemindert, was den „Umschaltvorgang“ weg von der zellulären Proliferation hin zur terminalen Differenzierung bewirkt.

\section{Fazit}

Xyloglucan scheint ein interessanter Naturstoff für die Dermatologie zu sein. Es konnte gezeigt werden, dass solche Gelbildner und Hydrokolloide nicht nur physikalisch Wunden abdecken, sondern auch pharmakodynamisch hochspezifisch in die Zellregulation eingreifen können.

Prof. Dr. Andreas Hensel, Münster

\section{Literatur}

Zacharski DM, Brandt S, Esch S, König S, Mormann M, Ulrich-Merzenich G, Hensel A. Xyloglucan from Tropaeolum majus seeds induces cellular differentiation of human keratinocytes by inhibition of EGFR phosphorylation and decreased activity of transcription factor CREB. Biomacromolecules 2015; 16: $2157-2167$ 